\title{
Un homme-orchestre dissonant
}

\author{
Daniel MAGGETTI et Dave LÜTHI \\ Université de Lausanne
}

\begin{abstract}
William Ritter (I867-I955) est un artiste aux multiples facettes : à la fois romancier, critique littéraire, critique musical, photographe, dessinateur, auteur de récits de voyage, il a produit une œuvre quantitativement imposante, que l'histoire littéraire de Suisse romande n'a pourtant que très partiellement prise en compte. Les articles réunis dans ce dossier entendent lever un coin du voile qui recouvre une figure dont les écrits, le parcours et les échanges intellectuels sont à même de nourrir une réflexion en profondeur sur les tendances de la culture de son temps.
\end{abstract}

Keywords: Suisse romande, Europe centrale, roman fin-de-siècle, wagnérisme, critique musicale, critique artistique

William Ritter (I867-I955) est une figure à la fois multiple, irritante et fascinante. Tour à tour ou simultanément, Ritter a été écrivain, essayiste, voyageur, critique musical, critique d'art, dessinateur, amateur de photographie ; catholique, il a grandi dans le cadre protestant du canton de Neuchâtel ; citoyen suisse, il a séjourné, parfois longuement, dans plusieurs capitales culturelles européennes, Paris, Munich, Vienne ; grand connaisseur des pays de l'Europe de l'Est, il a joué à l'égard de leur culture et de leur production artistique un rôle de passeur inégalé ; à une époque où la norme hétérosexuelle est socialement dominante et indiscutée, en particulier dans le contexte helvétique, il a manifesté ouvertement son homosexualité ; épistolier d'une fécondité à couper le souffle, il a dialogué avec des personnalités aux profils les plus variés, du Sar Joséphin Péladan à Le Corbusier. Mais s'il s'est inscrit dans la mouvance wagnérienne, puis a porté aux nues la musique de Mahler, faisant preuve parfois d'une réelle sensibilité pour les expressions d'avant-garde, Ritter a aussi souscrit à des positions idéologiques discutables de son temps, comme en témoigne la teneur antisémite de certains de ses propos. Sa trajectoire intellectuelle et artistique demeure elle-même inclassable, surtout si l'on tente de mettre en relation ses expériences cosmopolites d'avant la Première Guerre mondiale, et le repli qui caractérise la deuxième moitié de son existence, vécue dans le dénuement au bord d'un lac tessinois.

Volontiers excessif dans ses attitudes comme dans son discours et dans son style, ayant le dithyrambe aussi facile que l'éreintage sans appel, Ritter ne cesse de surprendre, tantôt par ses jugements à l'emporte-pièce - pas forcément dépourvus de fondement -, tantôt par sa manière particulière d'osciller entre le poncif et la plus vaste liberté de pensée, entre l'attachement 
à des visions surannées et l'attrait pour la nouveauté. Peu en phase avec le profil de l'intellectuel romand standard de son temps, William Ritter, en dépit de l'abondance de sa production, de la richesse de ses relations, de l'originalité de son parcours, a dès lors été négligé par la critique, notamment par les spécialistes de littérature suisse, qui ont fait l'impasse sur ce phénomène impossible à reconduire aux catégories d'analyse traditionnelles.

Régulièrement, pourtant, un coin du voile est levé, par des curieux que les fonds d'archives foisonnants de Berne, de La Chaux-de-Fonds et de Neuchâtel interpellent, ou par des spécialistes de domaines en apparence fort éloignés entre eux, dont Ritter se révèle être le point de convergence. Avec ce numéro de Versants, qui prolonge un séminaire de Master donné à l'université de Lausanne, en littérature et en histoire de l'art, au semestre d'automne 2017, nous avons voulu ajouter une pièce au dossier de la réception critique de Ritter, en mêlant les points de vue très autorisés des meilleurs connaisseurs de l'œuvre, les premières synthèses de quelques étudiants, les considérations issues de la pratique des archives. Au croisement de la littérature, de la musique, des arts plastiques, de la photographie, les contributions réunies ici ne sauraient prétendre à une quelconque exhaustivité. Notre souhait est plutôt qu'elles soient à même de stimuler l'intérêt des lecteurs pour les pousser à poursuivre leur découverte. Si ce but est atteint, nous aurons rempli notre rôle - en attendant qu'une recherche plus globale consacrée à Ritter voie le jour. 